\title{
Recent Epidemiology of Nonalcoholic Fatty Liver Disease
}

\author{
Soumya Murag ${ }^{1}$, Aijaz Ahmed², and Donghee Kim² \\ ${ }^{1}$ Department of Medicine, Santa Clara Valley Medical Center, Santa Clara, CA, and ${ }^{2}$ Division of Gastroenterology and Hepatology, \\ Stanford University School of Medicine, Stanford, CA, USA
}

\author{
Article Info \\ Received April 13, 2020 \\ Revised May 26, 2020 \\ Accepted May 26, 2020 \\ Published online September 15, 2020 \\ Corresponding Author \\ Donghee Kim \\ ORCID https://orcid.org/0000-0003-1919-6800 \\ E-mail dhkimmd@stanford.edu
}

\begin{abstract}
The ongoing obesity epidemic and the increasing recognition of metabolic syndrome have contributed to the growing prevalence of nonalcoholic fatty liver disease (NAFLD), the most common form of liver disease worldwide. It is imperative to understand the incidence and prevalence of NAFLD as it is associated with a profound economic burden of hospitalizations, including the shifting trends in liver transplantation. The long-term cumulative healthcare cost of NAFLD patients has been shown to be $80 \%$ higher than that of non-NAFLD patients. We explore diagnostic challenges in identifying those with NAFLD who have a higher predilection to progress to end-stage liver disease. We aim to assess all-cause and cause-specific mortality as it relates to NAFLD. (Gut Liver 2021;15:206-216)
\end{abstract}

Key Words: Fatty liver; Nonalcoholic steatohepatitis; Prevalence; Mortality; Natural history

\section{INTRODUCTION}

Fatty infiltration of the liver was only sporadically recognized in early literature and generally felt to be a benign condition. Until the 1990s, most studies recognized nonalcoholic fatty liver disease (NAFLD) as an "innocent bystander" rather than the "guilty party" in patients with cryptogenic cirrhosis, ${ }^{1}$ which was commonly used for end-stage liver disease in which the underlying etiology remains unidentified. The landmark comment by Ludwig et al. in 1980 first described the term "nonalcoholic steatohepatitis (NASH)" after identifying 20 nonalcoholic patients with liver biopsies showing changes similar to alcoholic hepatitis. Caldwell et al. ${ }^{3}$ recognized that the risk factors for cryptogenic cirrhosis paralleled those for NASH and NAFLD. Subsequent studies found that cirrhosis tends to occur at an older age in obese patients, further suggesting cryptogenic cirrhosis as a downstream effect of NASH and NAFLD. ${ }^{4}$

A major limitation in understanding the epidemiology of NAFLD has been the lack of a distinct classification of the disease. For example, under the International Classification of Diseases, Ninth Revision, Clinical Modification (ICD-9 CM), NAFLD has been grouped under cryptogenic cirrhosis or "other chronic nonalcoholic liver diseases" or "unspecified chronic liver disease without alcohol," underestimating at least $42.1 \%$ of individuals with NAFLD that were otherwise diagnosed by chart review. ${ }^{5}$ The misclassification of nomenclature has been an inherent problem in understanding pathophysiology and the prevalence of NAFLD, and its impact on downstream health outcomes. This review on NAFLD will further explore the current trends in epidemiology, recognize the importance of establishing the diagnosis of NAFLD in the context of chronic liver disease, and explore the diagnostic nuances in tracking the progression of NAFLD over time.

\section{DEFINITIONS}

The diagnosis of NAFLD requires more than or equal to $5 \%$ of hepatic fat accumulation and exclusion of other etiologies of liver disease such as viral hepatitis, autoimmune liver disease, hemochromatosis, Wilson's disease, drug-induced liver disease as well as significant alcohol consumption. ${ }^{6}$ The American Association for the Study of Liver Diseases Practice Guideline for NAFLD defines significant alcohol consumption as current or recent alcohol 
consumption of $>21$ standard drinks per week in men and $>14$ drinks per week in women over 2 year period. ${ }^{6}$ Diagnosis of NAFLD needs to be ruled out secondary causes of fatty infiltration, including lipodystrophy, starvation, Cushing's disease, and steatogenic medications (corticosteroids, amiodarone, methotrexate, tamoxifen, and antiretroviral therapy). ${ }^{6}$ As seen in Table 1, NAFLD refers to a broad array of histological variety from nonalcoholic fatty liver (NAFL) to NASH, which may or may not present with fibrosis that can progress to end-stage liver diseases such as cirrhosis or hepatocellular carcinoma (HCC). ${ }^{7} \mathrm{NASH}$ is defined as the presence of over $5 \%$ of hepatic fat accumulation and lobular inflammation with hepatocyte ballooning degeneration, with or without any fibrosis. ${ }^{6} \mathrm{NASH}$ cirrhosis is defined as the presence of cirrhosis with current or previous histological evidence of NAFL or NASH. ${ }^{6}$

\section{EPIDEMIOLOGY}

\section{Incidence of NAFLD}

There are scattered and limited data regarding the incidence of NAFLD in the general population. With the recent transition to ICD-10-CM with a specific diagnostic code for NAFLD, a study in England showed the incidence rate of NAFLD of 29 per 100,000 person-years with significant underestimated rates due to ICD-10 code. ${ }^{8} \mathrm{~A}$ meta-analysis published in 2016 showed that the pooled regional incidence rate estimates for Israel and Asia were 28.0 per 1,000 person-years ( $95 \%$ confidence interval [CI], 19.3 to 40.6$)$ and 52.3 per 1,000 person-years (95\% CI, 28.3 to 96.8 ) and respectively. ${ }^{9}$ A recent meta-analysis in Asia during 1999 to 2019, described the overall pooled incidence rate was 50.9 per 1,000 person-years $(95 \%$ CI, 44.8 to 57.4$).{ }^{10}$ Regarding trends in incidence of NAFLD, a community cohort study in Olmsted County, Minnesota, reported that NAFLD incidence increased 5-fold, from 62 per 100,000 person-years in 1997 to 329 per 100,000 person-years in 2014. ${ }^{11}$ The increase was highest (7-fold) in young adults aged $18-39$ years from 20 to 140 per 100,000 person-years. ${ }^{11}$ While NAFLD was identified based on the ICD-9-CM code-based algorithm and underestimated in this study, this study suggests a substantial increase in incidence, with a shift in the burden of disease toward a younger population. ${ }^{11}$

\section{Prevalence of NAFLD}

The true prevalence of NAFLD is hard to measure accurately due to the lack of consistent diagnostic criteria. NAFLD can be diagnosed using a radiologic assessment, while the degree of fibrosis and diagnosis of NASH requires a liver biopsy. The prevalence of NAFLD could be defined by histology, imaging, and blood tests, which are a much less reliable method of diagnosing NAFLD.

A systemic review and meta-analysis on the global epidemiology of NAFLD estimated prevalence of NAFLD by imaging to be $25.2 \%$ (95\% CI, 22.1 to 28.7$)^{9}$ with an estimated prevalence of NASH to be lower ranging from 3 to $5 \%{ }^{12}$ It is noted that the Middle East and South America have the highest prevalence of $32 \%$ (95\% CI, 13.5 to 58.2 ) and 30.5\% (95\% CI, 22.7 to 39.4), respectively, and the lowest prevalence in Africa at $13.5 \%$ (95\% CI, 5.9 to 28.7 ). ${ }^{9}, 13$ Table 2 summarizes the recent prevalence of NAFLD from across the world. ${ }^{14}$ Based on the United States (US) Third National Health and Nutrition Examination Survey (NHANES III), an extensive representative survey of the US civilian population, the prevalence of NAFLD by ultrasonography is estimated to be $34.0 \%$ in the US..$^{15}$ In the population-based cohort study from the United Kingdom, among 3,768 young adults (mean age, 24.0 years; interquartile range, 23.0 to 25.0) with available controlled attenuation parameter scores by transient elastography, 20.7\% (95\% CI, 19.4 to 22.0 ) subjects had suspected steatosis, with $10.0 \%$ presenting having severe steatosis. ${ }^{16}$ Interestingly, 2.7\% (95\% CI, 2.2 to 3.2) had suspected fibrosis (F2F4) by transient elastography. ${ }^{16}$

\section{Current trends and future projections in NAFLD}

Based on the serial NHANES dataset, the prevalence of NAFLD using noninvasive panels by US Fatty Liver Index, increased from $20.0 \%$ (1988-1994) to $28.3 \%$ (1999-2004) to $33.2 \%$ (2009-2012) and $31.9 \%$ (2013-2016) over 30 years. ${ }^{17}$ According to numerous studies which combined liver biopsy, noninvasive radiologic modalities as well as liver enzymes, $3 \%$ to $5 \%$ of NAFLD can progress to NASH with advanced fibrosis or cirrhosis, which is $1.25 \%$ of all

Table 1. Definition of Nonalcoholic Fatty Liver Disease

\begin{tabular}{ll}
\hline Nonalcoholic fatty liver disease & -Greater than $5 \%$ of hepatic fat accumulation \\
Nonalcoholic fatty liver & -Exclusion of other etiologies of liver diseases (i.e., infection, alcohol) \\
Nonalcoholic steatohepatitis (NASH) & -Hepatic steatosis without any histological manifestation of ballooning degeneration or fibrosis \\
& - Hepatic steatosis with histological manifestation of ballooning degeneration, lobular inflammation \\
NASH cirrhosis & wASH with the presence of cirrhosis
\end{tabular}


Table 2. Global Incidence and Prevalence of NAFLD

\begin{tabular}{|c|c|c|c|c|}
\hline Author (year) & Country & Description of study & Diagnostic method & Incidence or prevalence of NAFLD (\%) \\
\hline \multicolumn{5}{|l|}{ Incidence } \\
\hline $\begin{array}{l}\text { Younossi } \\
\text { et al. } \\
(2016)^{9}\end{array}$ & Asia and Israel & 5 Studies & $\begin{array}{l}\text { Ultrasonography, computed } \\
\text { tomography scan OR magnetic } \\
\text { resonance spectroscopy OR } \\
\text { blood testing }\end{array}$ & $\begin{array}{l}\text { For Asia, } 52.3 \text { per } 1,000 \\
\text { (95\% } \mathrm{Cl}, 28.31-96.77) \\
\text { Israel, } 28.01 \text { per } 1,000 \text { person-years } \\
\text { (95\% } \mathrm{Cl}, 19.34-40.57 \text { ) }\end{array}$ \\
\hline $\begin{array}{l}\text { Li et al. } \\
\qquad(2019)^{10}\end{array}$ & Asia & 18 Studies & $\begin{array}{l}\text { Ultrasonography, computed } \\
\text { tomography scan OR magnetic } \\
\text { resonance imaging/spectros- } \\
\text { copy OR liver biopsy OR blood } \\
\text { testing/predictive indices (fatty } \\
\text { liver index or hepatic steatosis } \\
\text { index) or ICD-9-CM codes }\end{array}$ & $\begin{array}{l}50.9 \text { per } 1,000 \text { person-years } \\
\quad(95 \% \mathrm{Cl}, 44.8-57.4)\end{array}$ \\
\hline $\begin{array}{l}\text { Allen et al. } \\
\qquad(2018)^{11}\end{array}$ & USA & $\begin{array}{l}\text { Community cohort study } \\
\text { ( } n=3,869 \text { subjects) }\end{array}$ & ICD-9-CM codes & 329 per 100,000 person-years in 2014 \\
\hline \multicolumn{5}{|l|}{ Prevalence } \\
\hline $\begin{array}{l}\text { Younossi } \\
\text { et al. } \\
(2016)^{9}\end{array}$ & $\begin{array}{l}\text { Africa, Asia, } \\
\text { Europe, } \\
\text { Middle } \\
\text { East, North } \\
\text { America, } \\
\text { South } \\
\text { America }\end{array}$ & $\begin{array}{l}86 \text { Studies included in meta- } \\
\text { analysis from } 22 \text { countries, } \\
1989-2015 \text { ( } n=8,515,431)\end{array}$ & $\begin{array}{l}\text { Ultrasonography, computed } \\
\text { tomography scan OR magnetic } \\
\text { resonance imaging/spectroscopy }\end{array}$ & $25.2 \%(95 \% \mathrm{Cl}, 22.1-27.9)$ \\
\hline $\begin{array}{l}\text { Li et al. } \\
\qquad(2019)^{10}\end{array}$ & Asia & $\begin{array}{l}237 \text { Observational studies } \\
\text { included in meta-analysis } \\
1999-2019 \text { ( } n=13,044,518)\end{array}$ & $\begin{array}{l}\text { Ultrasonography, computed } \\
\text { tomography scan OR magnetic } \\
\text { resonance imaging/spectros- } \\
\text { copy, serum based indicies, OR } \\
\text { liver biopsy }\end{array}$ & $30.5 \%(95 \% \mathrm{Cl}, 29.3-30.9)$ \\
\hline $\begin{array}{l}\text { Kim et al. } \\
\qquad(2013)^{15}\end{array}$ & USA & $\begin{array}{l}\text { US national representative } \\
\text { samples }(n=11,154)\end{array}$ & Ultrasonography & $\begin{array}{l}34.0 \% \\
3.2 \% \text { (advanced fibrosis) }\end{array}$ \\
\hline $\begin{array}{l}\text { Abeysekera } \\
\text { et al. } \\
(2020)^{16}\end{array}$ & UK & $\begin{array}{l}\text { UK Avon Longitudinal Study } \\
\text { of Parents and Children } \\
\text { (ALSPAC) cohort }(n=4,021)\end{array}$ & $\begin{array}{l}\text { Transient elastography with con- } \\
\text { trolled attenuation parameter } \\
\text { (CAP) score }\end{array}$ & $\begin{array}{l}20.7 \%(95 \% \mathrm{Cl}, 19.4-22.0) \text { for steatosis } \\
2.7 \%(95 \% \mathrm{Cl}, 2.2-3.2) \text { for suspected } \\
\quad \text { fibrosis (F2-F4) }\end{array}$ \\
\hline
\end{tabular}

NAFLD, nonalcoholic fatty liver disease; Cl, confidence interval; ICD-9-CM, International Classification of Diseases, Ninth Revision, Clinical Modification.

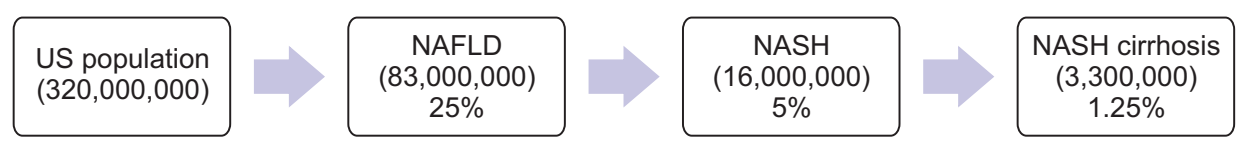

Fig. 1. Estimated number of individuals in the US population affected by nonalcoholic fatty liver disease (NAFLD). All numbers are estimated by the current prevalence of NAFLD, nonalcoholic steatohepatitis (NASH), NASH cirrhosis. ${ }^{9,12,18}$

population, ${ }^{12,18}$ as described in Fig. 1. The prevalence of NAFLD-related advanced fibrosis increased from 2.6\% (2005-2008) and 4.4\% (2009-2012) to 5.0\% (2013-2016) among subjects with NAFLD defined as the hepatic steatosis index; and from 3.3\% (2005-2008) and 6.4\% (20092012), to $6.8 \%$ (2013-2016) among those with NAFLD defined as US Fatty Liver Index $(\mathrm{p}<0.01) .{ }^{19}$ In this study, advanced fibrosis was defined as having at least one of the high probabilities for advanced fibrosis using three noninvasive fibrosis markers. ${ }^{19}$ Among type 2 diabetics from 1989 to 2018, the global prevalence was 55.5\% for NAFLD, $37.3 \%$ for NASH and $17.0 \%$ for advanced fibrosis. ${ }^{20} \mathrm{~A}$ recent study using the Markov prediction model reported that NAFLD is projected to increase by $21 \%$ from 83 million in 2015 to 101 million in $2030 .^{18} \mathrm{NASH}$ is forecasted to grow $63 \%$ from 17 million in 2015 to 27 million in $2030 .^{18}$ Incidence of decompensated cirrhosis is predicted to increase $168 \%$ by 2030 , while the incidence of HCC is projected to increase by $137 \% .{ }^{18}$ Using Markov modelling of the burden of NAFLD-related disease in eight countries including China, France, Germany, Italy, Japan, Spain, United Kingdom, and United States, prevalence of NASH will increase $15 \%$ to $56 \%$, while liver-related mortality and advanced liver disease will more than double due to an aging population and the projected rising prevalence of diabetes. ${ }^{21}$ 


\section{Hospitalizations and economic burdens in NAFLD}

The National Inpatient Sample is the largest publicly available, all-payer database of national hospital discharges in the US. It contains a $20 \%$ stratified and weighted sample of the US community and academic hospital. Hospitalization rates for decompensated cirrhosis and HCC increased approximately 1.5-fold from 2005-2006 to 2013-2014 in the US. ${ }^{22}$ Hospitalization rates with NAFLD-related decompensated cirrhosis increased from 13.4 per 100,000 hospitalizations to 32.1 per 100,000 hospitalizations with an annual increase of $10.6 \%$, a magnitude 2 -fold higher than chronic hepatitis $\mathrm{C}$ virus infection or alcoholic liver disease. ${ }^{22}$ The proportion of NAFLD among hospitalizations with decompensated cirrhosis steadily increased from $12.7 \%$ to $20.1 \%$ while the percentage of chronic hepatitis C infection (39.3\% to $27.6 \%$ ) and alcoholic liver disease decreased (39.0\% to 37.4\%) from 2005 to $2014 .{ }^{22}$ Hospitalizations for NAFLD-related HCC also increased with an annual rate of $8 \% .^{22}$ Other studies have also exhibited similar trends indicating NASH cirrhosis is the fastest-growing etiology of liver cirrhosis to contribute to hospitalizations. ${ }^{23}$ This trend echoes the idea that there is a subset of NAFLD patients who may be "rapid progressors" and need closer monitoring.

The burden of NAFLD on healthcare costs and resource utilization remains significant nowadays. A study based on real-world data from a US medical claims determined that the long-term cumulative healthcare cost of NAFLD is $80 \%$ higher than that of a non-NAFLD of similar age and metabolic comorbidities, ${ }^{24}$ although this study only considered private insurance and Medicare Advantage health plans. Patients limiting with healthcare access are less likely to have private insurance or Medicare Advantage plans and may be diagnosed with NAFLD at a later stage; there may be a considerable difference in healthcare costs for the management for these populations. ${ }^{25}$ This hypothesis raises the concern that NAFLD will impact minorities and patients who experience health disparities. ${ }^{25}$ A recent study in Sweden showed that healthcare costs were approximately twice as high in biopsy-confirmed NAFLD patients than in matched controls, which were primarily attributed to higher rates of hospitalizations and outpatient visits. ${ }^{26}$

\section{Risk factors and the extrahepatic manifestation of NAFLD}

Although NAFLD has been considered as the hepatic manifestation of the metabolic syndrome, a growing body of evidence suggests that NAFLD may be a key driver in metabolic syndrome. ${ }^{27}$ The hepatic manifestations of NAFLD are merely one component of a multi-organ systemic disease, which impacts on the cardiovascular, endo- crine, renal system, and extrahepatic malignancies. ${ }^{27}$ Type 2 diabetes or insulin resistance, dyslipidemia, and obesity are associated with the risk of NAFLD. ${ }^{27}$ Conditions with emerging associations are hypothyroidism, polycystic ovarian syndrome, obstructive sleep apnea, etc. ${ }^{27}$ Of these, obesity is the best documented as a risk factor for NAFLD. World Health Organization (WHO) Global Health Observatory data from 2014 estimates 15\% of women and $11 \%$ of men aged over 18 are obese, with many studies indicating a strong correlation between future prevalence of NAFLD and the increasing obesity epidemic. ${ }^{28} \mathrm{Com}$ pared with normal-weight counterparts, patients with compensated cirrhosis had worse outcomes if they were obese. ${ }^{29}$ While NAFLD is strongly associated with obesity, $3 \%$ to $30 \%$ of nonobese subjects had NAFLD, especially reported in Asian populations. ${ }^{30}$ In the US study based on the NHANES III, the prevalence of ultrasonography-detected hepatic steatosis to be $21 \%$ (mild-severe hepatic steatosis) in the lean population (body mass index $\left.<25 \mathrm{~kg} / \mathrm{m}^{2}\right)^{31}$ and $27 \%$ in nonobese population (body mass index $<30 \mathrm{~kg} / \mathrm{m}^{2}$ ). ${ }^{30}$ Potential risk factors for nonobese NAFLD include visceral obesity, high fructose intake, weight gain even within normal-weight limits, genetic risk factors. ${ }^{30} \mathrm{~A}$ recent study using the biopsy-proven NAFLD cohort determined that a strong association of NASH and significant fibrosis with the metabolically unhealthy phenotype irrespective of obesity status. ${ }^{32}$ The presence of NASH and significant fibrosis did not differ significantly between metabolic unhealthy nonobese and metabolic healthy obese groups. ${ }^{32}$ This has been thought to be due to metabolic milieu beyond obesity may play a pathogenic role.

Type 2 diabetes mellitus and insulin resistance promote lipolysis of the adipose tissue leading to deposition of the accumulation of free fatty acids into the liver. Individuals with diabetes had a 3-fold higher risk of death from NAFLD or NASH ${ }^{33}$ While the age-standardized mortality decreased in viral hepatitis, mortality increased rapidly in NAFLD at an annual rate of $11.6 \%$ (95\% CI, 9.5 to 13.8 ) among individuals with diabetes in the US from 2007 to $2017 .^{34}$ In fact, the association between diabetes and NAFLD is so strong, it is now questioned whether NAFLD is a pathogenic component to the development of type 2 diabetes. ${ }^{35}$ While the bidirectional relationship between NAFLD and type 2 diabetes is still being studied, it is well known that the presence of NAFLD in patients with type 2 DM seems to be a risk factor for all-cause mortality, as discussed below. ${ }^{36}$

Significant differences in the prevalence of NAFLD were observed between different race/ethnicity groups. Regardless of men and women, Hispanics had substantially higher NAFLD prevalence when compared to non- 
Hispanic whites and non-Hispanic blacks based on the third NHANES. ${ }^{37}$ A study using the recent 2011 to 2016 NHANES showed that prevalence was highest among Hispanic Americans (42.4\%), followed by non-Hispanic whites (28.4\%), Asian Americans (18.3\%) and nonHispanic blacks (17.4\%). ${ }^{38}$ Regarding advanced fibrosis, the NAFLD-related advanced fibrosis increased steadily in non-Hispanic whites. However, it leveled off during 2013 to 2016 in non-Hispanic blacks. ${ }^{19}$ Underlying genetic components are likely to play a role in the differences in the prevalence of NAFLD among race/ethnic groups. Genetic factors are further discussed below.

\section{MORTALITY IN NAFLD}

\section{All-cause mortality}

The first community-based study regarding the survival of patients $(n=435)$ diagnosed with NAFLD using imaging or histology was conducted in Olmsted County, Minnesota, from 1980 to 2000 . The study reported a significant decrease in survival for patients with NAFLD compared to the general Minnesota population of the same age and sex at 7.6 years of follow-up (standardized mortality ratio, 1.34; $95 \%$ CI, 1.003 to 1.76 ). ${ }^{39}$ Some of studies show similar findings with increased all-cause mortality with ranges of standardized mortality ratio of 1.34 to 2.6 and hazard ratio (HR) of 1.004 to $1.038 .{ }^{40}$ However, several other studies showed no difference in all-cause survival between subjects with or without NAFLD. ${ }^{40}$ A study using the third NHANES data with linked mortality data reported no significant difference in the all-cause mortality of USdiagnosed NAFLD compared with the non-NAFLD. ${ }^{15}$ The most important reasons for this inconsistency among studies might be due to diversity in the NAFLD spectrum according to the study population and consideration for metabolic abnormalities as confounders. A recent study using 27 years follow-up data of the third NHANES, NAFLD was associated with the increased risk for all-cause mortality (HR, 1.20; 95\% CI, 1.08 to 1.34 ), while this study did not consider any metabolic variables as confounders. In this study, the population attributable fraction of
NAFLD for all-cause mortality is 7.5\% (95\% CI, 3.0 to 12.0), while those of diabetes was $38.0 \%$ ( $95 \%$ CI, 13.1 to 63.0). ${ }^{41}$ This discrepancy sheds light on the importance of identifying high-risk populations within NAFLD that correlates with decreased survival. Compared to subjects without advanced fibrosis, those with a high probability of advanced fibrosis had a $69 \%$ increase in mortality after adjustment for other known predictors of mortality. ${ }^{15}$ These increases in mortality were almost entirely from cardiovascular causes. ${ }^{15}$ An international longitudinal study based on the liver biopsy determined that the fibrosis stage was independently associated with all-cause and liver-related mortality regardless of the presence or severity of other histologic features. ${ }^{42}$ In this study, the NAFLD scoring system did not provide any long-term prognostic information. ${ }^{42}$ Therefore, defining the presence of advanced fibrosis and the rate of fibrosis progression correlates with survival guides the development of diagnostic pathways that aim to stratify low-risk NAFLD patients from those that will progress to fibrosis or cirrhosis. While liver biopsy remains the gold standard, noninvasive fibrosis markers have been developed (Table 3), including fibrosis-4 (FIB-4), NAFLD fibrosis score as well as aspartate aminotransferase (AST) to platelet ratio index (APRI). A retrospective analysis using the NASH Clinical Research Network database validated the diagnostic performance of FIB-4 score (C-statistics: 0.80) and NAFLD fibrosis score (0.78) for advanced fibrosis. ${ }^{43}$ While stratification by fibrosis using NAFLD fibrosis score, FIB-4, and APRI at baseline proved to be a significant predictor of all-cause mortality, discussed previously, ${ }^{15}$ this study demonstrated that changes in FIB-4, APRI or NAFLD fibrosis score were significantly associated with disease progression including progression to advanced fibrosis. ${ }^{43}$ Further study is needed to confirm the longitudinal association between dynamic changes in noninvasive fibrosis panels and all-cause mortality in NAFLD. Similarly, vibration-controlled transient elastography and magnetic resonance elastography could identify advanced fibrosis with superior accuracy, ${ }^{44,45}$ however, limitation remains for clinical use for primary physician or epidemiologic study.

In addition to the fibrosis stage, several other factors

Table 3. Formulas of Noninvasive Fibrosis Marker Panels

\begin{tabular}{|c|c|}
\hline Formula & Equation \\
\hline Fibrosis-4 & $($ Age [years] $\times$ AST $[\mathrm{U} / \mathrm{L}]) /\left(\right.$ platelet $\left.\left[10^{9} / \mathrm{L}\right] \times(\mathrm{ALT}[\mathrm{U} / \mathrm{L}])^{1 / 2}\right)$ \\
\hline NAFLD fibrosis score & $\begin{array}{l}-1.675+(0.037 \times \text { age }[y e a r s])+\left(0.094 \times \mathrm{BMI}\left[\mathrm{kg} / \mathrm{m}^{2}\right]\right)+(1.13 \times \mathrm{IFG} \text { or diabetes }[\mathrm{yes}=1, \mathrm{no}=0]) \\
+(0.99 \times \text { AST/ALT })-\left(0.013 \times \text { platelet count }\left[10^{9} / \mathrm{L}\right]\right)-(0.66 \times \text { albumin }[\mathrm{g} / \mathrm{dL}])\end{array}$ \\
\hline APRI & ([AST/upper limit of normal]/platelet count $\left.\left[10^{9} / \mathrm{L}\right]\right) \times 100$ \\
\hline
\end{tabular}

AST, aspartate aminotransferase; ALT, alanine transferase; NAFLD, nonalcoholic fatty liver disease; BMI, body mass index; IFG, impaired fasting glucose; APRI, AST to platelet ratio index. 
should be considered in predicting outcomes of patients with NAFLD. First, the inclusion of age in the scoring systems may increase predictive power but create biases, especially in the setting of rising rates of childhood obesity. Secondly, race/ethnicity and genetic factors may play a pivotal role in all-cause mortality. A single variant of phos- pholipase domain-containing 3 or PNPLA3 (rs738409) gene was strongly associated with NAFLD, NASH, and decompensated cirrhosis. ${ }^{52,53}$ The highest frequency of this allele was in Hispanics, followed by non-Hispanic whites and least in non-Hispanic blacks. ${ }^{53}$ While we may be able to obtain static measurements of the presence or absence of

Loomba et al. Factors associated with histologic response in adult patients with nonalcoholic steatohepatitis.

Gastroenterology 2019;156:88-95

- Study design: 72 weeks, multicenter, randomized, placebo-controlled study by NASH Clinical Research Network (CRN) to assess $25 \mathrm{mg}$ obeticholic acid compared to placebo in NASH ( $\mathrm{n}=283)$. All had liver biopsy within 90 days of start and at end of study.

- Summary: Patients who had histological improvement had reductions in liver biochemistry at week 12 and 24 compared to those who did not achieve histological improvement.

- Risk factors: Baseline NAS, triglycerides, INR, AST and ALT reduction at week 24.

Jayakumar et al. Longitudinal correlations between MRE, MRI-PDFF, and liver histology in patients with non-alcoholic steatohepatitis: analysis of data from phase II trial of selonsertib. J Hepatol 2019;70:133-141

- Study design: Analysis of data from a multicenter phase II study of selonsertib to assess NASH patients with fibrosis stage 2 and 3 on liver biopsy. Pre and post treatment (24 weeks) assessments with magnetic resonance elastography (MRE), MR estimated proton density fat fraction (MRI-PDFF) were studied to assess correlation to histology on liver biopsy - Summary: MRE and MRI-PDFF both correlated with histology. $15 \%$ had fibrosis progression.

- Risk factors: Liver stiffness by MRE was significantly correlated with fibrosis stage as assessed by liver biopsy and non-invasive serum markers. Correlations at 24 weeks were more reliable than at baseline.

Sanyal et al. The natural history of advanced fibrosis due to nonalcoholic steatohepatitis: data from the simtuzumab trials. Hepatology 2019;70:1913-1927

- Study design: Analysis of data from two phase $2 b$, placebo-controlled trials of simtuzumab assessing predictors of fibrosis progression in patients with NASH and bridging cirrhosis.

- Summary: $22 \%$ of patients with baseline F3 fibrosis progressed to cirrhosis. Factors significantly associated with progression to cirrhosis included higher baseline values of and greater increases in hepatic collagen content, level of alpha-smooth muscle actin, and enhanced liver fibrosis score.

- Risk factors: Enhanced liver fibrosis score $\geq 9.8$, platelet count, FIB-4/NFS/APRI, platelet count predict progression to cirrhosis.

\section{Ajmera et al. Clinical utility of an increase in MRE in predicting fibrosis progression in NAFLD.}

Hepatology 2020;71:849-860

- Study design: Prospective longitudinal cohort study of biopsy proven NAFLD paired with MRE in 2011 and 2018.

- Summary: Liver stiffness on MRE had positive correlation with fibrosis stage. At follow up biopsy, $25 \%$ of patients without cirrhosis at baseline had fibrosis progression on histology. More than $15 \%$ increase in MRE was strongest predictor in progression to advanced fibrosis.

- Risk factors: Increase in MRE (liver stiffness measurement $\geq 15 \%$ ), AST, platelet count.

\section{Kleiner et al. Association of histological disease activity with progression of nonalcoholic fatty liver disease.}

JAMA Netw Open 2019;2:e1912565

- Study design: Prospective cohort substudy from NASH CRN who underwent 2 liver biopsies, at least 1 year apart

- Summary: $33.9 \%$ progressed by at least 1 stage of fibrosis. $16.8 \%$ showed progression from stage 0 to 2 on initial biopsy to stage 3 and 4 on follow up. This group were more likely to have metabolic syndrome.

- Risk factors: Baseline and change in AST, fibrosis stage, ballooning, portal inflammation, change in NAS.

Singh et al. Fibrosis progression in nonalcoholic fatty liver vs nonalcoholic steatohepatitis: a systematic review and meta-analysis of paired-biopsy studies. Clin Gastroenterol Hepatol 2015;13:643-654

- Study design: Systematic search of multiple databases, paired liver biopsy at least 1 year apart.

- Summary: $33.6 \%$ progressed by at least 1 stage of fibrosis. 1 stage of progression over 14.3 years for patients with NAFL and 7.1 years for patients with NASH.

- Risk factors: Presence of hypertension (OR 1.94; $95 \% \mathrm{Cl}, 1.00-3.74)$ and low AST: ALT ratio at the time of baseline biopsy.

Fig. 2. Recent literature summarizing risk factors for nonalcoholic fatty liver disease (NAFLD) progression. ${ }^{46-51}$

NASH, nonalcoholic steatohepatitis; NAS, NAFLD activity score; INR, international normalized ratio; AST, aspartate aminotransferase; ALT, alanine transferase; FIB-4, fibrosis-4; NFS, NAFLD fibrosis score; APRI, AST to platelet ratio index; OR, odds ratio; Cl, confidence interval. 
fibrosis, the level of disease activity is difficult to ascertain. Genetic variants may have underpinnings of understanding NAFLD disease activity. PNPLA3 and another common genetic variation called transmembrane 6 superfamily member 2 (TM6SF2) combined with lipoprotein insulin resistance index and age, were able to predict advanced fibrosis with a receiver operative characteristic curve of $0.82 .^{54}$ A recent population-based study showed that the homozygous PNPLA3 I148M (rs738409) GG genotype was longitudinally associated with the increased risk for all-cause mortality in the general population and NAFLD. ${ }^{55}$ Thirdly, not enough data exists to determine the frequency and duration of monitoring for NAFLD. A meta-analysis of patients with NASH with no fibrosis on biopsy indicates the mean rate of progression was 0.13 stage $(95 \% \mathrm{CI}, 0.07$ to 0.18 stage) per year. ${ }^{51}$ However, in subgroup analysis, $21.2 \%$ of patients progressed four stages of fibrosis over a mean $5.9( \pm 3.7)$ years. ${ }^{51}$ The heterogeneity in "rapid progressors" indicates a need for further investigation for factors that may determine fibrosis or all-cause mortality. Fig. 2 identifies risk factors such as fibrosis stage, biochemical markers, as well as imaging findings that predict the risk of progression to fibrosis and/or cirrhosis in recent literature. Overall, noninvasive fibrosis algorithms, platelet count, and AST have been consistently identified as predictors of fibrosis progression. The risk for progression of fibrosis in NAFLD consists of an interplay between genetic factors, biochemical markers, as well as intrinsic microbial factors. $^{56}$

In terms of mortality trends in chronic liver disease in the US, age-standardized hepatitis C virus infectionrelated mortality increased from 2007 to 2013, followed by a marked decrease after the introduction of directacting antiviral agents (from 2014 to 2016). ${ }^{57}$ The annual percentage changes (APC) in hepatitis $\mathrm{C}$ virus infectionrelated mortality increased $2.0 \%$ per year (2007-2014) but decreased 6.4\% per year (2014-2016). ${ }^{57}$ In contrast, agestandardized mortality increased for NAFLD (APC 6.1\% [2007-2013] and APC 11.3\% [2013-2016]). ${ }^{57}$ Mortality for cirrhosis (APC, 15.4\%; 95\% CI, 14.1 to 16.7) from NAFLD also increased over the previous decade. ${ }^{58}$ Fig. 3 shows an increase in the risk of all-cause mortality, with increasing stage of fibrosis among subjects with NAFLD irrespective of the presence of NASH. ${ }^{59}$ The magnitude of increasing relative risk appeared identical between subjects with NAFLD with/without NASH, with overlapping 95\% CI of relative risk estimates. ${ }^{59}$

\section{Cause-specific mortality and complications}

Cardiovascular disease is the leading cause of mortality in patients with NAFLD. Compared with other chronic liver diseases, the cause of death in NAFLD was more likely to be cardiovascular disease (approximately $20 \%){ }^{60}$ In addition, cardiovascular mortality is highest in NAFLD patients with metabolic syndromes. ${ }^{61}$ Traditional cardiovascular risk factors such as dyslipidemia, smoking, insulin resistance, hypertension, and abdominal obesity, share a substantial overlap with risk factors for NAFLD. ${ }^{62}$ Association between cardiovascular diseases and NAFLD cannot be attributable to shared risk factors between two diseases. ${ }^{63}$ Among patients with NAFLD, advanced fibrosis by noninvasive panels was a statistically significant predictor of cardiovascular mortality (HR, 3.46; 95\% CI, 1.91 to 6.25 for NAFLD fibrosis score; HR, 2.53; 95\% CI, 1.33 to 4.83 for APRI; HR, 2.68; 95\% CI, 1.44 to 4.99 for FIB-4). ${ }^{15}$ An international study with biopsy-proven NAFLD with bridging fibrosis or cirrhosis determined that NAFLD with bridging fibrosis had non-hepatic malignancies and car-

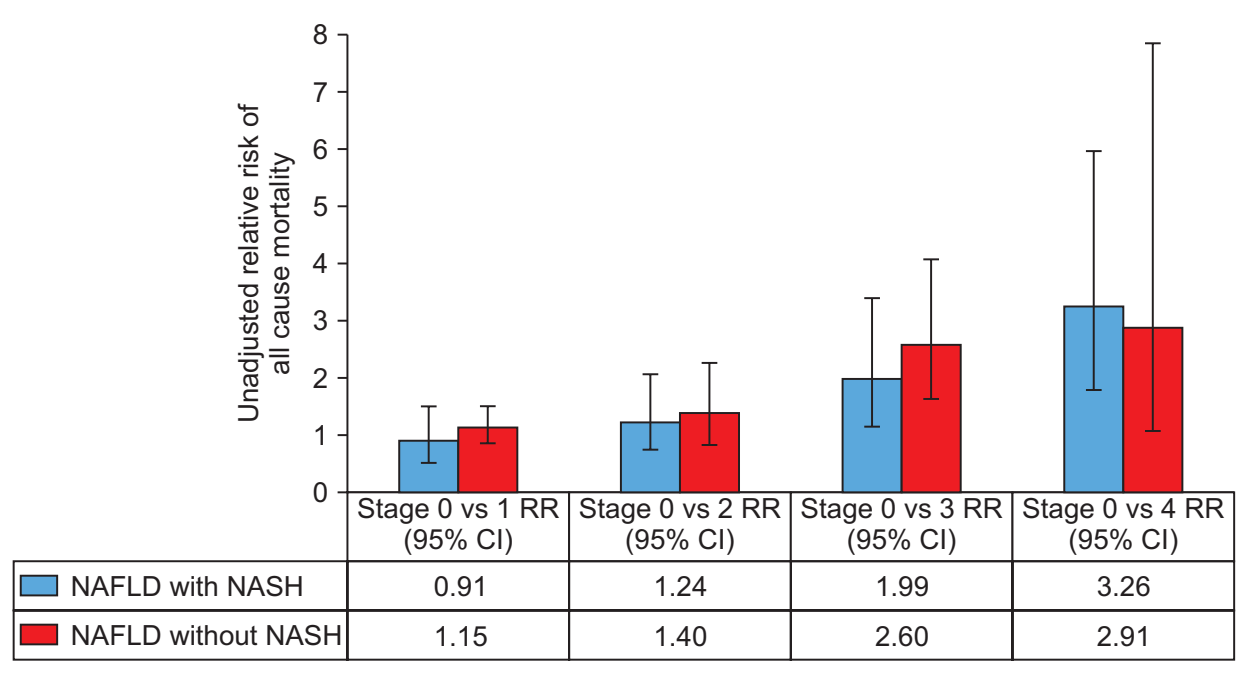

Fibrosis stage
Fig. 3. All-cause mortality of NAFLD patients with and without NASH by fibrosis stage. ${ }^{59}$ NAFLD, nonalcoholic fatty liver disease; NASH, nonalcoholic steatohepatitis; $\mathrm{RR}$, relative risk; $\mathrm{Cl}$, confidence interval. 
diovascular events predominantly, while NASH cirrhosis had mostly liver-related events. ${ }^{64}$ A meta-analysis including 34,043 adults showed that subjects with NAFLD more likely to develop non-fatal and/or fatal cardiovascular disease events than those without NAFLD (odds ratio, 1.64; $95 \%$ CI, 1.26 to 2.13 ) over a median 7 years. ${ }^{65}$ In addition, subjects with more severe NAFLD, including advanced fibrosis were had a higher risk of non-fatal and/or fatal cardiovascular events (odds ratio, 2.58 ; $95 \% \mathrm{CI}, 1.78$ to 3.75 ). ${ }^{65}$

The second most common cause of death is malignancy. Historical Korean cohort study including 25,947 subjects (NAFLD: $33.6 \%$ ) during the median 7.5 years follow-up reported that the cancer incidence rate of NAFLD was higher than that of non-NAFLD (782.9 vs 592.8 per 100,000 person-years: HR, 1.32; 95\% CI, 1.17 to 1.49$).{ }^{66} \mathrm{NAFLD}$ was associated with the incidence of colorectal cancer in males (HR, 2.01; 95\% CI, 1.10 to 3.68) and breast cancer in females (HR, 1.92; 95\% CI, 1.15 to 3.20). ${ }^{66}$ Another cohort study from Olmsted County, Minnesota, demonstrated that NAFLD was associated with a nearly 2 -fold increase in the overall risk of incident cancers during a median 8 years (incidence rate ratio, 1.9; 95\% CI, 1.3 to 2.7 ). ${ }^{67}$ The highest risk increase was noted in uterine, followed by stomach, pancreas, and colon cancer. ${ }^{67}$

Liver disease is also an essential contributor to death among patients with NAFLD, being the third most common cause and accounting for $13 \%$ of all deaths in a study by Adams et al..$^{39}$ In contrast, "chronic liver disease and cirrhosis" is the 13th leading cause of death among the Minnesota general population, accounting for $<1 \%$ of all deaths. This implies that the increased overall mortality rate among NAFLD patients compared with the general population is at least partly due to complications of NAFLD ${ }^{39}$ Due to second-generation direct-acting antiviral agents approved late 2013, there has been a significant reduction in the waitlist burden related to chronic hepatitis $\mathrm{C}$ virus infection; however, the number of total registrants awaiting liver transplantation continues to rise. In 2016, for the first time, both alcoholic liver disease and NASH had surpassed chronic hepatitis $\mathrm{C}$ virus infection as the leading indications of liver transplantation in the US. ${ }^{68} \mathrm{HCC}$ is the third leading cause of cancer-related deaths in the world. There is an increase in the rate of HCC reported by Surveillance, Epidemiology, and End Results-Medicare linked database from 2004 to 2009, which is not explained by the increase of incidence in NAFLD. Data suggest a poorer prognosis with HCC from NAFLD-cirrhosis as $62 \%$ of patients with NAFLD-related HCC died within 1 year than those with HCC related to viral hepatitis and a majority (84.3\%) of patients with NAFLD-related HCC died of their primary liver cancer. ${ }^{69} \mathrm{~A}$ study using the US national mortality data showed that there was a linear increase in the age-standardized HCC-related mortality rates for NAFLD (19.1\%; 95\% CI, 14.0 to 24.5$){ }^{58}$ Among patients with NAFLD, cardiovascular disease increased at a gradual pace (APC, $2.0 \%$; 95\% CI, 0.6 to 3.4), whereas liver-related mortality increased rapidly (APC, 12.6\%; 95\% CI, 11.7 to 13.5) based on the US national mortality data from 2007 to $2017 .^{60}$

\section{CURRENT HEALTH POLICY}

Even though the prevalence of NAFLD is already $30 \%$ and projected to increase substantially, there is no clear consensus currently on the most cost-effective to identify the approximately 100 million NAFLD patients in the US general population that will progress to the $1.5 \%$ who experience fibrosis and cirrhosis. In fact, no clear national policies exist regarding identifying high-risk populations and monitoring for progression to cirrhosis. In 2018 and 2019, a comprehensive survey of the national policy in 29 European countries showed an absence of written national strategies or action plans for NAFLD and a small portion of countries had national clinical guidelines (34\%) and recommended screening for NAFLD (38\%) in all patients with either diabetes, obesity, and/or metabolic syndrome. ${ }^{70}$ This could indicate the lack of appreciation for the high prevalence and the lack of clear guidelines in identifying and following high-risk patients using current diagnostic methods and classification scores.

\section{CONCLUSIONS}

This review summarizes the worldwide incidence and prevalence of NAFLD, discusses diagnostic nuances in determining subjects with NAFLD who progress to cirrhosis, and quantifies downstream impacts on the healthcare economy in the context of all-cause and cause-specific mortality. Currently, the estimated prevalence of NAFLD by imaging was $25.2 \%$, with an estimated prevalence of NASH to be lower, ranging from $3 \%$ to $5 \%$. NAFLD can progress to NASH with advanced fibrosis or cirrhosis in about $3 \%$ to $5 \%$ of NAFLD. The fibrosis stage is independently associated with all-cause and liver-related mortality regardless of the presence or severity of other histologic features. Further study is needed to confirm the longitudinal association between dynamic changes in noninvasive fibrosis panels and all-cause mortality. Age, race/ethnicity, sex, metabolic comorbidities, and genetics were associated with outcomes of NAFLD. Cardiovascular disease, extra- 
hepatic malignancy, and end-stage liver disease are the leading cause of mortality in patients with NAFLD. This review identifies that screening guidelines for NAFLD to identify high-risk populations and monitoring for progression to cirrhosis remains a significant unmet need in the field.

\section{CONFLICTS OF INTEREST}

No potential conflict of interest relevant to this article was reported.

\section{ORCID}

Soumya Murag https://orcid.org/0000-0002-7548-2281 Aijaz Ahmed https://orcid.org/0000-0002-3609-8586 Donghee Kim https://orcid.org/0000-0003-1919-6800

\section{REFERENCES}

1. Day CP, James OF. Hepatic steatosis: innocent bystander or guilty party? Hepatology 1998;27:1463-1466.

2. Ludwig J, Viggiano TR, McGill DB, Oh BJ. Nonalcoholic steatohepatitis: Mayo Clinic experiences with a hitherto unnamed disease. Mayo Clin Proc 1980;55:434-438.

3. Caldwell SH, Oelsner DH, Iezzoni JC, Hespenheide EE, Battle EH, Driscoll CJ. Cryptogenic cirrhosis: clinical characterization and risk factors for underlying disease. Hepatology 1999;29:664-669.

4. Ratziu V, Bonyhay L, Di Martino V, et al. Survival, liver failure, and hepatocellular carcinoma in obesity-related cryptogenic cirrhosis. Hepatology 2002;35:1485-1493.

5. Corey KE, Kartoun U, Zheng H, Shaw SY. Development and validation of an algorithm to identify nonalcoholic fatty liver disease in the electronic medical record. Dig Dis Sci 2016;61:913-919.

6. Chalasani N, Younossi Z, Lavine JE, et al. The diagnosis and management of nonalcoholic fatty liver disease: practice guidance from the American Association for the Study of Liver Diseases. Hepatology 2018;67:328-357.

7. Angulo P. Nonalcoholic fatty liver disease. N Engl J Med 2002;346:1221-1231.

8. Whalley S, Puvanachandra P, Desai A, Kennedy H. Hepatology outpatient service provision in secondary care: a study of liver disease incidence and resource costs. Clin Med (Lond) 2007;7:119-124.

9. Younossi ZM, Koenig AB, Abdelatif D, Fazel Y, Henry L, Wymer M. Global epidemiology of nonalcoholic fatty liver
disease-Meta-analytic assessment of prevalence, incidence, and outcomes. Hepatology 2016;64:73-84.

10. Li J, Zou B, Yeo YH, et al. Prevalence, incidence, and outcome of non-alcoholic fatty liver disease in Asia, 1999-2019: a systematic review and meta-analysis. Lancet Gastroenterol Hepatol 2019;4:389-398.

11. Allen AM, Therneau TM, Larson JJ, Coward A, Somers VK, Kamath PS. Nonalcoholic fatty liver disease incidence and impact on metabolic burden and death: a 20 year-community study. Hepatology 2018;67:1726-1736.

12. Vernon G, Baranova A, Younossi ZM. Systematic review: the epidemiology and natural history of non-alcoholic fatty liver disease and non-alcoholic steatohepatitis in adults. Aliment Pharmacol Ther 2011;34:274-285.

13. Perumpail BJ, Khan MA, Yoo ER, Cholankeril G, Kim D, Ahmed A. Clinical epidemiology and disease burden of nonalcoholic fatty liver disease. World J Gastroenterol 2017;23:8263-8276.

14. Muthiah MD, Sanyal AJ. Burden of disease due to nonalcoholic fatty liver disease. Gastroenterol Clin North Am 2020;49:1-23.

15. Kim D, Kim WR, Kim HJ, Therneau TM. Association between noninvasive fibrosis markers and mortality among adults with nonalcoholic fatty liver disease in the United States. Hepatology 2013;57:1357-1365.

16. Abeysekera KWM, Fernandes GS, Hammerton G, et al. Prevalence of steatosis and fibrosis in young adults in the UK: a population-based study. Lancet Gastroenterol Hepatol 2020;5:295-305.

17. Younossi ZM, Stepanova M, Younossi Y, et al. Epidemiology of chronic liver diseases in the USA in the past three decades. Gut 2020;69:564-568.

18. Estes C, Razavi H, Loomba R, Younossi Z, Sanyal AJ. Modeling the epidemic of nonalcoholic fatty liver disease demonstrates an exponential increase in burden of disease. Hepatology 2018;67:123-133.

19. Kim D, Kim W, Adejumo AC, et al. Race/ethnicity-based temporal changes in prevalence of NAFLD-related advanced fibrosis in the United States, 2005-2016. Hepatol Int 2019;13:205-213.

20. Younossi ZM, Golabi P, de Avila L, et al. The global epidemiology of NAFLD and NASH in patients with type 2 diabetes: a systematic review and meta-analysis. J Hepatol 2019;71:793-801.

21. Estes C, Anstee QM, Arias-Loste MT, et al. Modeling NAFLD disease burden in China, France, Germany, Italy, Japan, Spain, United Kingdom, and United States for the period 2016-2030. J Hepatol 2018;69:896-904.

22. Kim D, Cholankeril G, Li AA, et al. Trends in hospitalizations for chronic liver disease-related liver failure in the United States, 2005-2014. Liver Int 2019;39:1661-1671. 
23. Axley P, Ahmed Z, Arora S, et al. NASH is the most rapidly growing etiology for acute-on-chronic liver failure-related hospitalization and disease burden in the United States: a population-based study. Liver Transpl 2019;25:695-705.

24. Allen AM, Van Houten HK, Sangaralingham LR, Talwalkar JA, McCoy RG. Healthcare cost and utilization in nonalcoholic fatty liver disease: real-world data from a large U.S. claims database. Hepatology 2018;68:2230-2238.

25. Yoo ER, Ahmed A, Kim D. Economic burden and healthcare utilization in nonalcoholic fatty liver disease. Hepatobiliary Surg Nutr 2019;8:181-183.

26. Hagström H, Nasr P, Ekstedt M, et al. Health care costs of patients with biopsy-confirmed nonalcoholic fatty liver disease are nearly twice those of matched controls. Clin Gastroenterol Hepatol 2020;18:1592-1599.

27. Li AA, Ahmed A, Kim D. Extrahepatic manifestations of nonalcoholic fatty liver disease. Gut Liver 2020;14:168-178.

28. Fan JG, Kim SU, Wong VW. New trends on obesity and NAFLD in Asia. J Hepatol 2017;67:862-873.

29. Berzigotti A, Garcia-Tsao G, Bosch J, et al. Obesity is an independent risk factor for clinical decompensation in patients with cirrhosis. Hepatology 2011;54:555-561.

30. Kim D, Kim WR. Nonobese fatty liver disease. Clin Gastroenterol Hepatol 2017;15:474-485.

31. Kim D, Kim W. Non-overweight fatty liver disease (NOFLD): a distinct entity? Hepatology 2012;56(S1):886A-887A.

32. Kim D, Kim W, Joo SK, et al. Association between body sizemetabolic phenotype and nonalcoholic steatohepatitis and significant fibrosis. J Gastroenterol 2020;55:330-341.

33. Byrne CD, Targher G. NAFLD: a multisystem disease. J Hepatol 2015;62(1 Suppl):S47-S64.

34. Kim D, Cholankeril G, Kim SH, Abbasi F, Knowles JW, Ahmed A. Increasing mortality among patients with diabetes and chronic liver disease from 2007 to 2017. Clin Gastroenterol Hepatol 2020;18:992-994.

35. Tilg H, Moschen AR, Roden M. NAFLD and diabetes mellitus. Nat Rev Gastroenterol Hepatol 2017;14:32-42.

36. Anstee QM, Targher G, Day CP. Progression of NAFLD to diabetes mellitus, cardiovascular disease or cirrhosis. Nat Rev Gastroenterol Hepatol 2013;10:330-344.

37. Lazo M, Hernaez R, Eberhardt MS, et al. Prevalence of nonalcoholic fatty liver disease in the United States: the Third National Health and Nutrition Examination Survey, 19881994. Am J Epidemiol 2013;178:38-45.

38. Golabi P, Paik J, Hwang JP, Wang S, Lee HM, Younossi ZM. Prevalence and outcomes of non-alcoholic fatty liver disease (NAFLD) among Asian American adults in the United States. Liver Int 2019;39:748-757.

39. Adams LA, Lymp JF, St Sauver J, et al. The natural history of nonalcoholic fatty liver disease: a population-based cohort study. Gastroenterology 2005;129:113-121.
40. Kwak MS, Kim D. Long-term outcomes of nonalcoholic fatty liver disease. Curr Hepatol Rep 2015;14:69-76.

41. Alvarez CS, Graubard BI, Thistle JE, Petrick JL, McGlynn KA. Attributable fractions of NAFLD for mortality in the United States: results from NHANES III with 27 years of follow-up. Hepatology 2020;72:430-440.

42. Angulo P, Kleiner DE, Dam-Larsen S, et al. Liver fibrosis, but no other histologic features, is associated with long-term outcomes of patients with nonalcoholic fatty liver disease. Gastroenterology 2015;149:389-397.

43. Siddiqui MS, Yamada G, Vuppalanchi R, et al. Diagnostic accuracy of noninvasive fibrosis models to detect change in fibrosis stage. Clin Gastroenterol Hepatol 2019;17:18771885.

44. Kim D, Kim WR, Talwalkar JA, Kim HJ, Ehman RL. Advanced fibrosis in nonalcoholic fatty liver disease: noninvasive assessment with MR elastography. Radiology 2013;268:411-419.

45. Siddiqui MS, Vuppalanchi R, Van Natta ML, et al. Vibrationcontrolled transient elastography to assess fibrosis and steatosis in patients with nonalcoholic fatty liver disease. Clin Gastroenterol Hepatol 2019;17:156-163.

46. Loomba R, Sanyal AJ, Kowdley KV, et al. Factors associated with histologic response in adult patients with nonalcoholic steatohepatitis. Gastroenterology 2019;156:88-95.

47. Jayakumar S, Middleton MS, Lawitz EJ, et al. Longitudinal correlations between MRE, MRI-PDFF, and liver histology in patients with non-alcoholic steatohepatitis: analysis of data from a phase II trial of selonsertib. J Hepatol 2019;70:133-141.

48. Sanyal A, Harrison SA, Ratziu V, et al. The natural history of advanced fibrosis due to nonalcoholic steatohepatitis: data from the simtuzumab trials. Hepatology 2019;70:1913-1927.

49. Ajmera VH, Liu A, Singh S, et al. Clinical utility of an increase in magnetic resonance elastography in predicting fibrosis progression in nonalcoholic fatty liver disease. Hepatology 2020;71:849-860.

50. Kleiner DE, Brunt EM, Wilson LA, et al. Association of histologic disease activity with progression of nonalcoholic fatty liver disease. JAMA Netw Open 2019;2:e1912565.

51. Singh S, Allen AM, Wang Z, Prokop LJ, Murad MH, Loomba R. Fibrosis progression in nonalcoholic fatty liver vs nonalcoholic steatohepatitis: a systematic review and metaanalysis of paired-biopsy studies. Clin Gastroenterol Hepatol 2015;13:643-654.

52. Mandorfer M, Scheiner B, Stättermayer AF, et al. Impact of patatin-like phospholipase domain containing 3 rs738409 G/G genotype on hepatic decompensation and mortality in patients with portal hypertension. Aliment Pharmacol Ther 2018;48:451-459.

53. Romeo S, Kozlitina J, Xing C, et al. Genetic variation in 
PNPLA3 confers susceptibility to nonalcoholic fatty liver disease. Nat Genet 2008;40:1461-1465.

54. Danford CJ, Connelly MA, Shalaurova I, et al. A pathophysiologic approach combining genetics and insulin resistance to predict the severity of nonalcoholic fatty liver disease. Hepatol Commun 2018;2:1467-1478.

55. Wijarnpreecha K, Scribani M, Raymond P, et al. PNPLA3 gene polymorphism and overall and cardiovascular mortality in the United States. J Gastroenterol Hepatol 2020;35:17891794.

56. Jayakumar S, Loomba R. Review article: emerging role of the gut microbiome in the progression of nonalcoholic fatty liver disease and potential therapeutic implications. Aliment Pharmacol Ther 2019;50:144-158.

57. Kim D, Li AA, Gadiparthi C, et al. Changing trends in etiology-based annual mortality from chronic liver disease, from 2007 through 2016. Gastroenterology 2018;155:1154-1163.

58. Kim D, Li AA, Perumpail BJ, et al. Changing trends in etiology-based and ethnicity-based annual mortality rates of cirrhosis and hepatocellular carcinoma in the United States. Hepatology 2019;69:1064-1074.

59. Taylor RS, Taylor RJ, Bayliss S, et al. Association between fibrosis stage and outcomes of patients with nonalcoholic fatty liver disease: a systematic review and meta-analysis. Gastroenterology 2020;158:1611-1625.

60. Kim D, Adejumo AC, Yoo ER, et al. Trends in mortality from extrahepatic complications in patients with chronic liver disease, from 2007 through 2017. Gastroenterology 2019;157:1055-1066.

61. Younossi ZM, Otgonsuren M, Venkatesan C, Mishra A. In patients with non-alcoholic fatty liver disease, metabolically abnormal individuals are at a higher risk for mortality while metabolically normal individuals are not. Metabolism 2013;62:352-360.
62. Marchesini G, Bugianesi E, Forlani G, et al. Nonalcoholic fatty liver, steatohepatitis, and the metabolic syndrome. Hepatology 2003;37:917-923.

63. Stepanova M, Younossi ZM. Independent association between nonalcoholic fatty liver disease and cardiovascular disease in the US population. Clin Gastroenterol Hepatol 2012;10:646-650.

64. Vilar-Gomez E, Calzadilla-Bertot L, Wai-Sun Wong V, et al. Fibrosis severity as a determinant of cause-specific mortality in patients with advanced nonalcoholic fatty liver disease: a multi-national cohort study. Gastroenterology 2018;155:443457.

65. Targher G, Byrne CD, Lonardo A, Zoppini G, Barbui C. Non-alcoholic fatty liver disease and risk of incident cardiovascular disease: a meta-analysis. J Hepatol 2016;65:589-600.

66. Kim GA, Lee HC, Choe J, et al. Association between nonalcoholic fatty liver disease and cancer incidence rate. J Hepatol 2018;68:140-146.

67. Allen AM, Hicks SB, Mara KC, Larson JJ, Therneau TM. The risk of incident extrahepatic cancers is higher in non-alcoholic fatty liver disease than obesity: a longitudinal cohort study. J Hepatol 2019;71:1229-1236.

68. Cholankeril G, Ahmed A. Alcoholic liver disease replaces hepatitis $\mathrm{C}$ virus infection as the leading indication for liver transplantation in the United States. Clin Gastroenterol Hepatol 2018;16:1356-1358.

69. Younossi ZM, Otgonsuren M, Henry L, et al. Association of nonalcoholic fatty liver disease (NAFLD) with hepatocellular carcinoma (HCC) in the United States from 2004 to 2009. Hepatology 2015;62:1723-1730.

70. Lazarus JV, Ekstedt M, Marchesini G, et al. A cross-sectional study of the public health response to non-alcoholic fatty liver disease in Europe. J Hepatol 2020;72:14-24. 